ARTICLE

Received 15 Dec 2014 | Accepted 15 Sep 2016 | Published 10 Nov $2016 \quad$ DOl: 10.1038/ncomms13261

\title{
Hydrogenated monolayer graphene with reversible and tunable wide band gap and its field-effect transistor
}

Jangyup Son ${ }^{1}$, Soogil Lee ${ }^{1}$, Sang Jin Kim², Byung Cheol Park ${ }^{3}$, Han-Koo Lee ${ }^{4}$, Sanghoon Kim, Jae Hoon Kim³, Byung Hee Hong ${ }^{2} \&$ Jongill Hong ${ }^{1}$

Graphene is currently at the forefront of cutting-edge science and technology due to exceptional electronic, optical, mechanical, and thermal properties. However, the absence of a sizeable band gap in graphene has been a major obstacle for application. To open and control a band gap in functionalized graphene, several gapping strategies have been developed. In particular, hydrogen plasma treatment has triggered a great scientific interest, because it has been known to be an efficient way to modify the surface of single-layered graphene and to apply for standard wafer-scale fabrication. Here we show a monolayer chemical-vapourdeposited graphene hydrogenated by indirect hydrogen plasma without structural defect and we demonstrate that a band gap can be tuned as wide as $3.9 \mathrm{eV}$ by varying hydrogen coverage. We also show a hydrogenated graphene field-effect transistor, showing that on/off ratio changes over three orders of magnitude at room temperature.

\footnotetext{
${ }^{1}$ Department of Materials Science and Engineering, Yonsei University, 50 Yonsei, Seodaemun, Seoul 03722, Korea. ${ }^{2}$ Department of Chemistry, Seoul National University, Seoul 08826, Korea. ${ }^{3}$ Department of Physics, Yonsei University, Seoul 03722, Korea. ${ }^{4}$ Pohang Accelerator Laboratory, Pohang 37673 , Korea. Correspondence and requests for materials should be addressed to J.H. (email: hong.jongill@yonsei.ac.kr).
} 
G raphene has opened a new arena in the field of twodimensional condensed matter physics since its first isolation from graphite by means of micromechanical exfoliation ${ }^{1-3}$. Based on fascinating properties, graphene provides a radically new platform for future electronic and photonic science and technology ${ }^{4-8}$. Despite extraordinary physical and chemical properties of graphene, the absence of a sizeable band gap has delayed envisioned graphene-based electronic and photonic science and technology. A number of research groups have taken up the challenge of opening a band gap in graphene and they have mostly followed two main routes: (1) forming graphene nanoribbons ${ }^{9,10}$ having a few nanometres in width and (2) a chemical modification of the graphene surface ${ }^{11}$. The graphene nanoribbon has demonstrated its tunability of a magnitude of the band gap and its capability of switchable nano-devices. What makes it challenging is, however, that it is not easy to control the width of ribbons on the order of a nanometre. In contrast, the chemical approach is more practical for largescale productions and industrial applications. It has also been known to open a band gap of $\sim 4.0 \mathrm{eV}$ according to theoretical calculations $^{12,13}$. Among various chemical approaches, researches on opening the band gap of graphene by hydrogenation have been intensively studied. Not only theoretical works on hydrogenated graphene ${ }^{12,13}$ but many experimental efforts on hydrogenation techniques such as a hydrogen-plasma treatment $^{14}$, a reaction with hydrogen atoms in a hydrogen silsesquioxane (HSQ) film ${ }^{15,16}$ and an exposure of atomic hydrogen (deuterium) beams ${ }^{17}$ have been reported. In particular, hydrogenplasma treatment has been regarded as an efficient way to modify the surface of graphene and to apply for a standard wafer-scale fabrication. However, the energetic ions have etched carbon atoms and induced structural damage in graphene. Despite various experimental efforts ${ }^{9,14-19}$, none of them has succeeded in observing a sizable band gap of at least $\geq 1 \mathrm{eV}$ or in managing a device operation yet. The absence of a sizeable band gap in graphene synthesized by the present technologies and experimental challenges originating from the intrinsic nature and extrinsic degradation of graphene are impeding further developments in envisioned carbon-based electronics.

Here, we show that monolayer graphene hydrogenated by indirect hydrogen plasma at room temperature acquires a band gap up to $\sim 4.0 \mathrm{eV}$ that can be tuned by varying hydrogen coverage while preserving the structural integrity of the original graphene. We first demonstrate that the intrinsic band-gapped graphene we created fully functions as a field-effect transistor (FET), showing that conductivity at gate voltages changes over three orders of magnitude at room temperature.

\section{Results}

Determination of a band gap by transport measurements. Opening a band gap in chemical-vapour-deposited (CVD) graphene $^{20,21}$ was possible by exposing pristine graphene to indirect (or remote) hydrogen plasma in vacuum at room temperature. Synchrotron radiation X-ray photoemission spectroscopy (PES) showed that the valence band of hydrogenated graphene was shifted down to energy much lower than that of graphene or $\mathrm{Au}$, as shown in Fig. 1a. (We hereafter refer to hydrogenated monolayer graphene as $\mathrm{H}-\mathrm{Gr}$.) The magnitude of the shift increased and then saturated at $3.5 \mathrm{eV}$ (green arrow in Fig. 1) as the exposure time to the hydrogen plasma or hydrogen coverage $\eta$ on graphene increased. We found that the hydrogen coverage $\eta$ saturated at $\sim 25 \%$ when we further increased the exposure time (see Supplementary Fig. 1 and Supplementary Note 1). When the downshift of the valence-band maximum was $1.7 \mathrm{eV}$ (blue arrow in Fig. 1) and $3.5 \mathrm{eV}$, the $\eta$ for $\mathrm{H}-\mathrm{Gr}$ was $\sim 12 \%$ and $\sim 25 \%$, respectively. Small shifts of $\sim 0.4 \mathrm{eV}$ (black arrow in Fig. 1) were observed in the conduction-band minimum by near-edge X-ray absorption fine structure (NEXAFS) spectroscopy, but these shifts did not change significantly with the coverage. The resulting band gap of CVD graphene was opened as a result of exposure to indirect hydrogen plasma ranging from 2.1 to $3.9 \mathrm{eV}$.

A close investigation of these spectra reveals that the band-gap opening indeed arises from the hydrogenation of graphene. The $\pi$ and the $\pi^{*}$ orbitals, a fingerprint of graphene, disappeared and were significantly reduced, respectively, with the coverage. In other words, the $s p^{2}$ orbitals rehybridized with the local $s p^{3}$ orbitals as a result of hydrogenation. In addition, the $\mathrm{C}^{\star}=\mathrm{O}$ orbital, located at $\sim 3.2 \mathrm{eV}$ above the $\pi^{\star}$ orbital, also disappeared as the hydrogenation continued, indicating that the hydrogen replaces oxygen chemisorbed on the CVD graphene. It is noteworthy that the pronounced $\pi^{*}$ band signifies topological ripples and corrugations, and that the intermediate states between $\pi^{*}$ and $\sigma^{\star}$ contain residual adsorbates commonly observed in CVD graphene ${ }^{22}$. Such defects are extrinsic and are inevitably induced during the fabrication process of CVD graphene $e^{20,21,23}$.

Our in-situ heating PES measurements of $\mathrm{H}-\mathrm{Gr}$ directly prove that our indirect hydrogen plasma indeed inflicts minimal damage on graphene, that is, that $\mathrm{H}-\mathrm{Gr}$ has no significant defects in the honeycomb basal plane after hydrogenation. (We provide additional evidence for this later.) The indirect plasma technique is well known to significantly reduce damages to a surface, because the surface is not in direct contact with the plasma, which contains energetic particles. Both the band gaps of $\mathrm{H}-\mathrm{Gr}$ created at an $\eta$ of $12 \%$ and $25 \%$, for example, returned to 0 (Fig. 1b) when $\mathrm{H}-\mathrm{Gr}$ was heated in the PES chamber $\left(<8 \times 10^{-10}\right.$ Torr $)$ at $550 \mathrm{~K}$ for $2 \mathrm{~h}$. Besides, the recovery of the $\pi$ orbital below the Fermi energy proves that hydrogen that had bonded with the $p_{\mathrm{z}}$-orbital of carbon was desorbed during heating and that $\mathrm{H}-\mathrm{Gr}$ was reduced to metallic graphene. Such reversibility has been observed only when the graphene framework was not seriously damaged by surface treatments. Our observation of the full recovery of $\mathrm{H}-\mathrm{Gr}$ to graphene bears a critical implication for the modulation of a band gap of graphene: we can control the band gap either by exposing the graphene to indirect hydrogen plasma or by thermal treatment of the resulting $\mathrm{H}-\mathrm{Gr}$.

Optical absorption experiments. Not only the transport bandgap measurements (PES and NEXAFS spectroscopy) but also optical absorption experiments support the wide tunability of a band gap of graphene induced by our plasma treatment. Initially, the absorption spectra of graphene acquired from near-infrared to ultraviolet at room temperature in air exhibited a general feature of a $\pi$ plasmon located at $\sim 4.6 \mathrm{eV}$ due to excitonic Fano resonance ${ }^{24}$. As hydrogenation proceeded, the $\pi$ plasmon peak is blue-shifted by up to $\sim 0.04 \mathrm{eV}$ (Fig. 2a). The optical bandwidth of $\mathrm{H}-\mathrm{Gr}$ (as estimated from the higher-lying absorption background) ranged from 1.4 to $4.6 \mathrm{eV}$, depending on the hydrogen coverage $\eta$ (Supplementary Fig. 2 and Supplementary Note 2), which is different from the bandwidth measured with the synchrotron radiation X-ray source. However, it was also saturated at $\eta$ of $25 \%$. It is not uncommon to see such a difference in the magnitude of the bandwidth estimated by the two measurements: whereas the transport measurement integrates both indirect and direct excitations about the Fermi energy, the optical absorption is mainly due to direct (vertical) band-to-band excitation. Therefore, the optical spectra often reveal the upper bound of the bandwidth in the case of indirect band-gap semiconductors. This is most likely the case in what we observed here-more of which later. Meanwhile, it is worth noting that the 

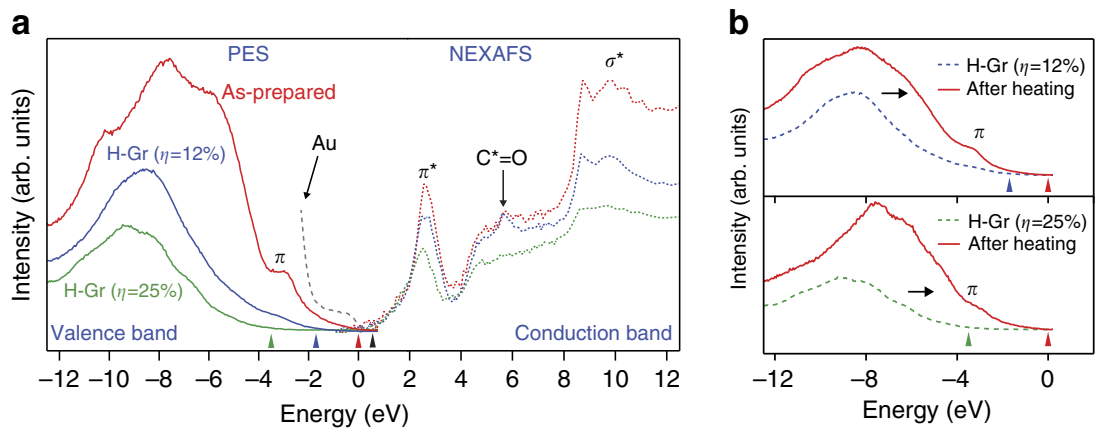

Figure 1 | Evolution of the electronic band structure of hydrogenated monolayer graphene (H-Gr) at $\mathbf{3 0 0} \mathbf{K}$. (a) PES and NEXAFS spectra of graphene and $\mathrm{H}-\mathrm{Gr}$. Red, blue and green arrows indicate the valence-band maximum of as-prepared graphene, $\mathrm{H}-\mathrm{Gr}(\eta=12 \%)$ and $\mathrm{H}-\mathrm{Gr}(\eta=25 \%)$, respectively. Black arrow means the shift of the conduction band of both $\mathrm{H}-\mathrm{Gr}(\eta=12 \%)$ and $\mathrm{H}-\mathrm{Gr}(\eta=25 \%)$. The band gap was tunable up to $3.9 \mathrm{eV}$ by the change of hydrogen coverage $\eta$. (b) Recovery of the $\pi$ orbital of graphene in PES spectra after annealing of $\mathrm{H}-\mathrm{Gr}$ in vacuum at $550 \mathrm{~K}$ for $2 \mathrm{~h}$.
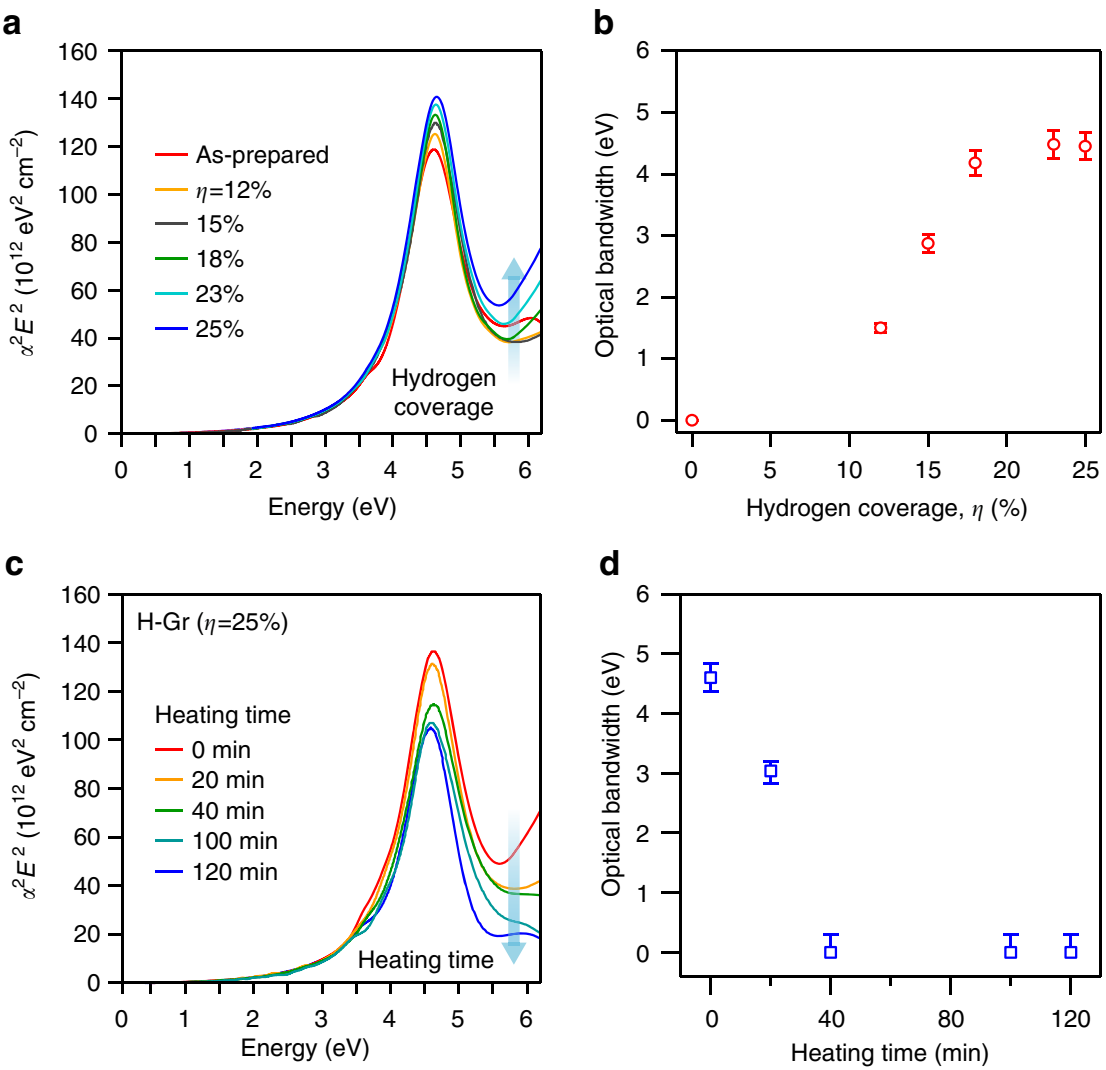

Figure 2 | Optical bandwidth and reversibility of H-Gr at $\mathbf{3 0 0} \mathbf{K}$. (a,b) The evolution of optical (near infrared, visible and ultraviolet) absorption spectra of graphene and $\mathrm{H}-\mathrm{Gr}$ and their estimated bandwidths as a function of hydrogen coverage $\eta$. In the figure, $\alpha$ is the optical absorption coefficient and $E$ is the incident photon energy. The zero optical bandwidth indicates the screening of interband tails by exciton resonance. (c,d) The full recovery of the optical absorption spectra and bandwidths of $\mathrm{H}-\mathrm{Gr}(\eta=25 \%)$ retuning to the pristine graphene state after thermal treatment at $550 \mathrm{~K}$ under Ar atmosphere as a function of annealing time. The error bars correspond to $\pm 0.5 \%$ uncertainty in the measurement of optical transmission acquired by spectrophotometry.

crucial reversible recovery from $\mathrm{H}-\mathrm{Gr}$ to graphene was repeatedly confirmed through ex-situ optical absorption experiments. After we annealed $\mathrm{H}-\mathrm{Gr}$ with an optical bandwidth of $4.6 \mathrm{eV}$ at $550 \mathrm{~K}$ for $20 \mathrm{~min}$ under Ar atmosphere and carried out optical absorption experiments, the optical bandwidth decreased from 4.6 to $3.0 \mathrm{eV}$ (Fig. 2c,d). The longer thermal treatment accelerated dehydrogenation of $\mathrm{H}-\mathrm{Gr}$ and restored $\mathrm{H}-\mathrm{Gr}$ to the pristine state of graphene as indicated by the disappearance of the absorption background.
Structural integrity. We found that the hydrogenation or the formation of $s p^{3} \mathrm{C}-\mathrm{H}$ bonds by indirect hydrogen plasma occurred without significant damage to its carbon network. As the hydrogen coverage increased, the Raman spectra evolved in a distinct manner, as shown in Fig. 3a. Although the G and 2D peaks decreased with the coverage, the $\mathrm{D}$ and $\mathrm{D}^{\prime}$ peaks started to appear $^{25-27}$ and then became dominant, which is known to be caused by defects such as $s p^{3} \mathrm{C}-\mathrm{H}$ bonds and/or the broken symmetry of a carbon $s p^{2}$ network ${ }^{14}$. Our Raman spectra also 
a
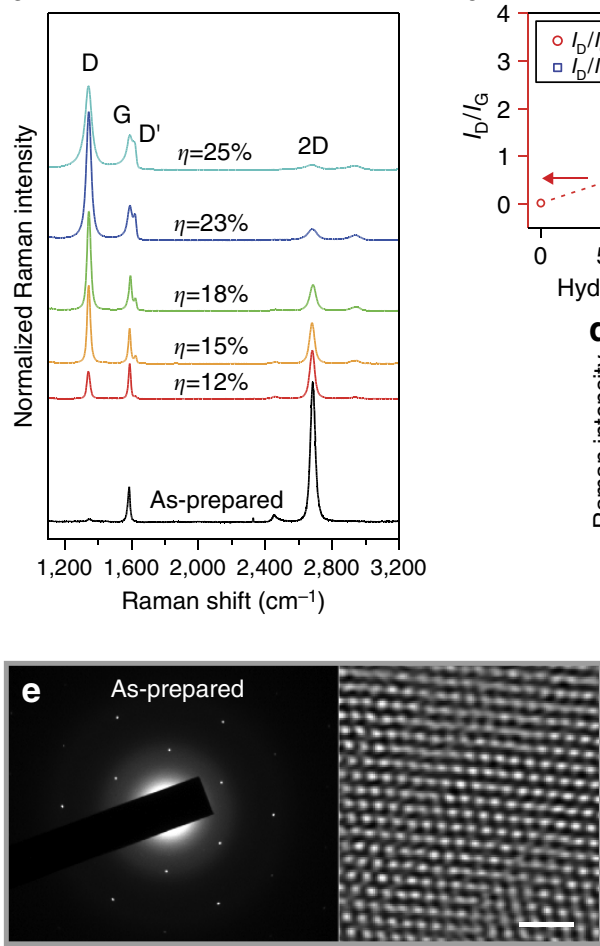

d b

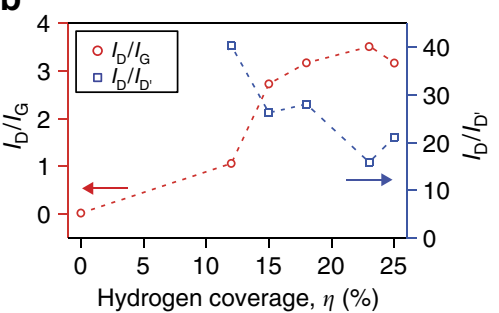

C

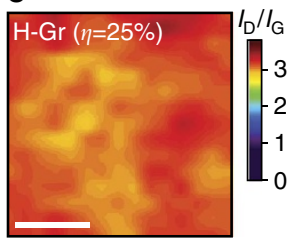

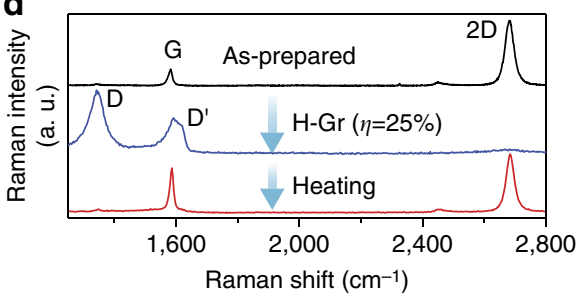

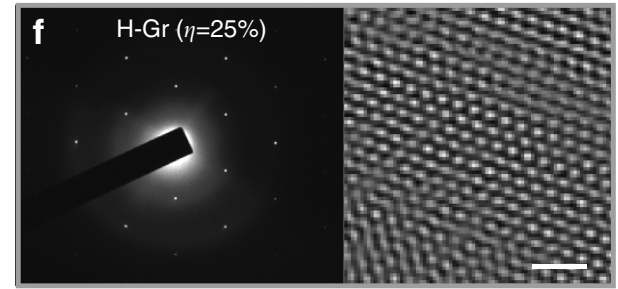

Figure 3 | Raman behaviours and atomic images of graphene and H-Gr. (a)The Raman changes of H-Gr with an increase in hydrogen coverage $\eta$ at $300 \mathrm{~K}$. (b) The ratio of integrated intensity, $I_{\mathrm{D}} / I_{\mathrm{G}}$ and $I_{\mathrm{D}} / I_{\mathrm{D}^{\prime}}$, as a function of hydrogen coverage $\eta$. (c) Raman mapping of $I_{\mathrm{D}} / I_{\mathrm{G}}$ for $\mathrm{H}-\mathrm{Gr}$ at $\eta=25 \%$. Scale bar, $5 \mu \mathrm{m}$. (d) The recovery of Raman spectra of $\mathrm{H}$-Gr treated by ex-situ heating for the optical absorption experiments. (e,f) Selected area electrondiffraction patterns and high-resolution transmission electron microscopy images for graphene and $\mathrm{H}-\mathrm{Gr}$, respectively. Scale bar, $1 \mathrm{~nm}$.

reveal two additional features (Fig. 3b). One is that the ratio of the integrated intensity of the $\mathrm{D}$ peak to that of the $\mathrm{G}$ peak $\left(I_{\mathrm{D}} / I_{\mathrm{G}}\right)$ gradually increased ${ }^{27}$ and saturated at $\sim 3.0$ with the coverage of up to $25 \%$. It is noteworthy that the ratio mapping indicated a uniform distribution of the defects throughout the specimen (Fig. 3c). The ratio of the integrated intensity of the $\mathrm{D}$ peak to that of the $\mathrm{D}^{\prime}$ peak $\left(I_{\mathrm{D}} / I_{\mathrm{D}^{\prime}}\right)$ showed a similar saturation tendency and stayed at over 16, never dropping after saturation. The former ratio and its mapping imply that the defects are uniformly distributed and saturated at $\eta$ of $25 \%$, as the distance between defects no longer increases, and the latter ratio shows that the defect is not an atomic-vacancy type but an $s p^{3}$ type in $\mathrm{H}-\mathrm{Gr}^{28}$.

The possibility of broken symmetry of the honeycomb $s p^{2}$ network can be ruled out by high-resolution transmission electron microscopy and by the evidence of the return of $\mathrm{H}-\mathrm{Gr}$ to the pristine graphene state by the aforementioned thermal treatment. Neither structural damage nor disintegration of the innate symmetry of graphene was found in the micrographs (Fig. 3f). The sixfold symmetric dots shown in the electrondiffraction patterns also support the existence of a high-quality honeycomb network for both graphene and $\mathrm{H}-\mathrm{Gr}$. The $\mathrm{H}-\mathrm{Gr}$ treated by ex-situ heating for the optical absorption experiments fully recovered the Raman features of graphene (Fig. 3d). The most surprising discovery was that the $\mathrm{D}$ peak had completely disappeared after heating. Such Raman behaviours are impossible to observe, unless graphene maintains a two-dimensional carbon network of high quality. Typical damages found in hydrogenated graphene by plasma treatment could be amorphization and void formation, and they render the graphene irreversibly hydrogenated, making it unusable for long-term applications.
Electrical transport measurements and FET. The temperature (T) dependence of resistivity $(\rho)$ of our H-Gr shows that the band gap stems from a long-range order at an $\eta$ of $25 \%$, whereas it can be opened by delocalized defect states at the lower hydrogen coverages (Supplementary Fig. 3 and Supplementary Note 3). As shown in Fig. $4 \mathrm{a}$, the $\rho-1 / \mathrm{T}$ of $\mathrm{H}-\mathrm{Gr}(\eta=25 \%)$ showed three distinct behaviours: (1) intrinsic excitation, (2) variable-range hopping and (3) saturation towards the Mott minimum conductivity-all of which are essential features observed in crystal semiconductors $^{29}$. It is noteworthy that amorphous or disordered gapped materials such as graphene oxide cannot show such distinct stages ${ }^{30}$. In fact, the band-gap curve-fitted by the relation of $\left.\rho=\rho_{0} \exp \left[E_{\mathrm{g}} /\left(2 k_{\mathrm{B}} T\right)\right]\right)$ in the intrinsic region was $\sim 3.8 \mathrm{eV}$, which is close to the band gap $(\sim 3.9 \mathrm{eV})$ measured by transport band-gap measurements. On the other hand, when hydrogenation coverage was insufficient, the $\rho-1 / T$ showed the behaviour of variable-range hopping conductance, in which $\rho$ is proportional to $T^{-1 / 3}$ (not shown here) as others observed ${ }^{14}$.

Judging from the size of a band gap and the temperaturedependent resistivity of our $\mathrm{H}-\mathrm{Gr}$ showing an $\eta$ of $25 \%$, we have reached the conclusion that our saturated $\mathrm{H}-\mathrm{Gr}$ can have a form of para-type $\mathrm{C}_{4} \mathrm{H}$ (refs 31-33), a single-sided hydrogenated graphene. As a matter of fact, hydrogenation in the $\mathrm{H}-\mathrm{Gr}$ happens most probably on the top surface of graphene transferred onto a $\mathrm{SiO}_{2} / \mathrm{Si}$ substrate, because our hydrogen atoms do not have strong enough energy to penetrate graphene due to the mild and non-destructive nature of indirect hydrogen plasma. According to the electronic band structure calculation based on density functional theory $32,33, \mathrm{C}_{4} \mathrm{H}$-type hydrogenated graphene has an indirect band gap of $3.5 \mathrm{eV}$. This is close to the $3.9 \mathrm{eV}$ that we observed through transport measurements, which provide 
a Intrinsic b

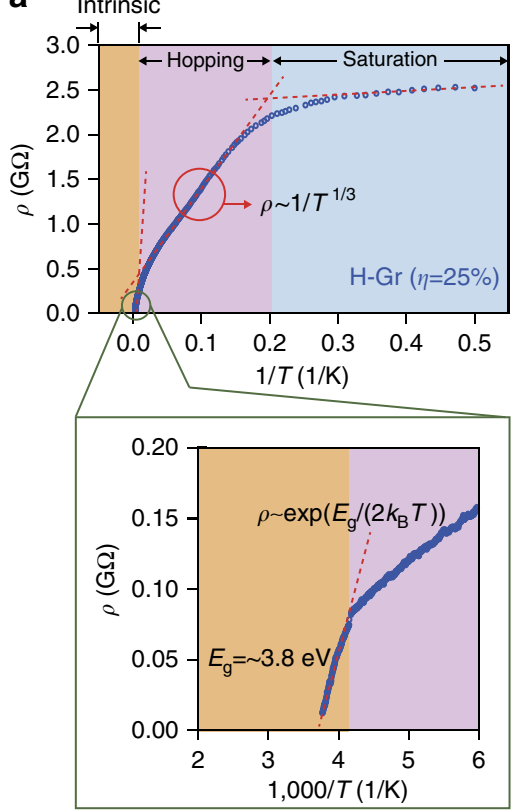

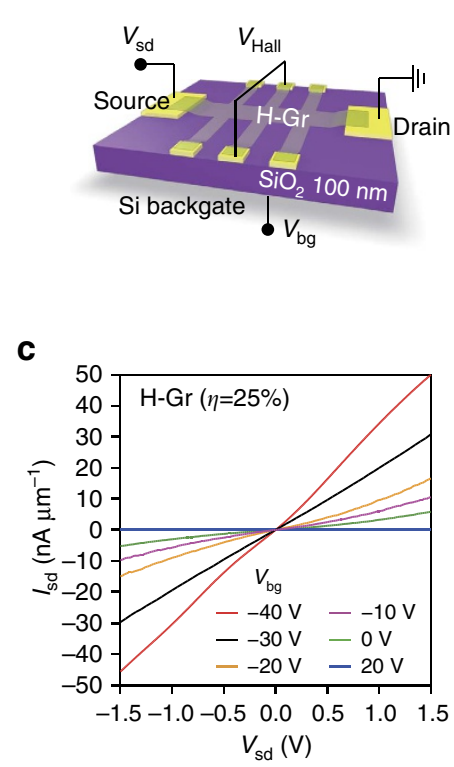

d

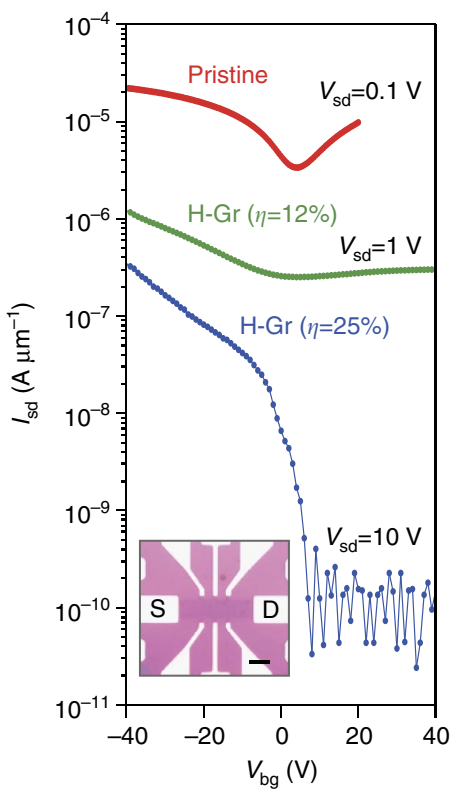

Figure 4 | Temperature ( $(\boldsymbol{T})$-dependent resistivity $(\boldsymbol{\rho})$ of $\mathbf{H}-\mathbf{G r}$ and the characteristics of $\mathbf{H}$-Gr's FETs at $\mathbf{3 0 0} \mathbf{K}$. (a) The behaviour of $\rho-1 / T$ of $\mathrm{H}-\mathrm{Gr}$ $(\eta=25 \%)$ reveals the prevailing transport mechanism as crystal semiconductors. (b) Schematics of H-Gr's FETs and its experimental setup. (c) $I-V$ sourcedrain characteristics were initially nonlinear but became linear as the gate bias was increased from -40 to $20 \mathrm{~V}$. (d) Change in current on the sweep of a back-gate voltage (inset: optical image of the devices; scale bar, $10 \mu \mathrm{m}$ ).

integrated signals about the Fermi energy. It is worth noting that the density functional theory calculation usually underestimates the value of the band gap. The different magnitudes of the band gaps we measured by X-ray and optical absorption add further weight to our conclusion. The wide bandwidth of $\sim 4.5 \mathrm{eV}$ estimated by optical absorption is believed to be the result of a direct (vertical) band-to-band excitation: optical absorption has been found to be very sensitive to the direct transitions at highsymmetry points, such as $K, M$ and $G$, in the Brillouin zone of $\mathrm{C}_{4} \mathrm{H}$-type hydrogenated graphene. The difference in the magnitude of the measured band gaps may also in part stem from the difference in the degree of hydrogenation or in the distribution of $\mathrm{C}-\mathrm{H}$ bonds possibly caused by the different substrates used for transport measurements and optical absorption measurements$\mathrm{Si} / \mathrm{SiO}_{2}$ and sapphire, respectively (see Methods).

Finally, we demonstrate the functionality of a FET made from $\mathrm{H}-\mathrm{Gr}$ (Fig. 4b-d). Our proof-of-concept FET made out of hydrogenated monolayer graphene with a band gap of $3.9 \mathrm{eV}$ on $100 \mathrm{~nm}$-thick $\mathrm{SiO}_{2}$ gate dielectric showed a dramatic change in current of over $10^{3}$ on/off ratio on the sweep of a back-gate voltage at room temperature as shown in Fig. $4 \mathrm{~d}$. To the best of our knowledge, this is the highest ratio achieved among functionalized graphene-based FETs ever reported and is an additional clear evidence for the intrinsic band gap of our $\mathrm{H}-\mathrm{Gr}$ being the result of the hybridization of the periodic structure. However, our on/off ratio could not go over $10^{4}$. We believe such a limit can be ascribed to the high off-current due to leakage current through the thermally oxidized $\mathrm{SiO}_{2}$ of poor quality. $I-V$ source-drain characteristics were initially nonlinear but became linear as the gate bias was increased over $-20 \mathrm{~V}$ (Fig. $4 \mathrm{c}$, as similarly observed in a wide-band gap FET such as fluorinated graphene $\mathrm{FET}^{34}$ and large band gap semiconductor devices ${ }^{35}$. This indicates that the electrical transport process has changed from tunnelling through Schottky contact between metal and $\mathrm{H}-\mathrm{Gr}$ to resistive conduction due to excitation of electrons into the conduction band of the hydrogenated graphene. When the positive gate bias was increased up to $40 \mathrm{~V}$, the off-current state was maintained at the noise level, that is, it was clearly off-state at gate bias exceeding positive $5 \mathrm{~V}$. Such asymmetric conductance response to the gate bias indicates that our hydrogenated graphene is a $p$-type semiconductor. The increase of the band gap inevitably leads to a decrease in mobility due to the dominant formation of an $s p^{3}$ bond ${ }^{36}$. For example, the mobility was $\sim 4 \mathrm{~cm}^{2} \mathrm{~V}^{-1} \mathrm{~s}^{-1}$ for the FET with a band gap of $3.9 \mathrm{eV}$ (Supplementary Table 1 and Supplementary Note 4). To show that the mobility can be further increased by improving our primitive proof-of-concept structure and minimizing the substrate effect, we fabricated several other FETs. A top-gated FET with polymethyl methacrylate (PMMA) $/ \mathrm{Al}_{2} \mathrm{O}_{3}$ dielectric, for example, exhibited more than double the mobility $\left(\sim 9 \mathrm{~cm}^{2} \mathrm{~V}^{-1} \mathrm{~s}^{-1}\right)$, indicating that the performance of FETs can be significantly improved by implementing a high- $\kappa$ gate dielectric and an improved FET structure (Supplementary Fig. 4). We note that our mobility value is significantly lower than the mobility of pristine graphene but is still higher than that of current FETs for flexible electronics, for which graphene is one of the most suitable materials because of its superb mechanical ${ }^{37}$ and optical properties ${ }^{7}$, and large area synthesis technique ${ }^{20,21}$. In the case of the FET made out of H-Gr with a band gap of $1.7 \mathrm{eV}$ $(\eta \sim 12 \%)$, the on/off ratio was unexpectedly low. This is because the band gap was opened by random hydrogenation ${ }^{31}$, which is evidenced by the temperature-dependent resistivity (Supplementary Fig. 3 and Supplementary Note 3 ).

The very considerable advantage of our method is that a device can be built entirely of graphene: a conductor, that is, graphene, and a semiconductor and an insulator, that is, our hydrogenated graphene with small and large band gaps ${ }^{38}$. Moreover, $\mathrm{H}-\mathrm{Gr}$ in this study promise not only to elucidate many controversial theoretical predictions but also to ignite intense research in the field of semiconducting graphene, as our new band-gap engineering protocol for hydrogenated graphene will play a critical role in realizing a broad range of novel applications 
encompassing integrated carbon electronics, optoelectronics and photonics.

In summary, we have demonstrated that the indirect exposure of CVD graphene to hydrogen plasma successfully modulated the band gap of graphene and, as a result, opened a band gap as wide as $\sim 4.0 \mathrm{eV}$ without breaking the honeycomb structural symmetry of graphene; we have also shown that our hydrogenated graphene can fully function as a FET at room temperature. All of our hydrogenated graphene with a band gap retained very high structural integrity and was fully reduced to pristine graphene with a zero band gap simply by heating. Our study of monolayer graphene hydrogenated by indirect hydrogen plasma promises to provide answers to many controversial theoretical predictions and to trigger studies on new aspects of semiconducting graphene that have so far been hampered by experimental difficulties. Thus, this new form of graphene should play a significant role in enabling unique applications for long-envisioned carbon-based electronics and photonics.

\section{Methods}

Preparation of monolayer CVD grapheme. Graphene was prepared under high vacuum by CVD on Cu foils and transferred onto a heavily $p$-doped Si substrate with $300 \mathrm{~nm}$-thick $\mathrm{SiO}_{2}$ for Raman, X-ray photoemission spectroscopy, PES and NEXAFS measurements. A sapphire substrate was used and CVD graphene showing $\sim 2.3 \%$ absorption in the visible range was chosen for hydrogenation and optical absorption measurements.

Hydrogenation. Hydrogen plasma was generated in a separate gun with a microwave power of $180 \mathrm{~W}$ at $2.5 \mathrm{GHz}$. The flow rate of hydrogen was 25 s.c.c.m. and the working pressure was $10 \mathrm{mtorr}$. The as-prepared CVD graphene was downstream positioned $10 \mathrm{~cm}$ away from the end of the gun.

Device fabrication and electrical measurements. At first, to fabricate graphenebased FETs, electrodes were patterned by a lift-off process after $\mathrm{Cr} / \mathrm{Pt}(5 / 25 \mathrm{~nm})$ was sputter-deposited on the patterns prepared by photolithography. Then, CVD graphene was transferred onto a heavily $p$-doped $\mathrm{Si}$ substrate with $100 \mathrm{~nm}$-thick $\mathrm{SiO}_{2}$. PMMA/HSQ bilayer resist on graphene was exposed by an electron beam and HSQ-uncovered PMMA/graphene layers were etched out by $\mathrm{O}_{2}$ plasma after developing. After removing them in acetone, FETs with graphene was obtained. They were annealed at $300^{\circ} \mathrm{C}$ for $1 \mathrm{~h}$ in a gas mixture of $\mathrm{Ar} / \mathrm{H}_{2}$ (10:1) to remove residual contaminations. Finally, FETs were treated by hydrogen plasma. We measured the electrical properties at various back-gate bias from $2 \mathrm{~K}$ to $300 \mathrm{~K}$ in vacuum. For a two-probe measurement, we applied constant source-drain voltage $\left(V_{\mathrm{sd}}\right)$ and FET mobility was extracted from the differential curve of the electric field effect. For a four-probe Hall measurement, constant source-drain current of $100 \mathrm{nA}$ was applied and the Hall voltage $\left(V_{\mathrm{H}}\right)$ was measured. The mobility and carrier density were estimated from $V_{\mathrm{H}}$ measured by the four-probe Hall measurement.

\section{Optical band-gap measurements and Raman spectroscopy. The optical} transmission and absorption coefficient of graphene and $\mathrm{H}-\mathrm{Gr}$ were measured at room temperature via visible-ultraviolet spectrophotometry (Cary 5G, Varian Inc.) over the wavelength range of $175-3,300 \mathrm{~nm}$ at normal incidence. Raman spectra were obtained with a Horiba Jobin-Yvon Lab Ram HR spectrometer with an Ar laser wavelength of $514.5 \mathrm{~nm}$ at room temperature. Diffraction patterns and atomic images were taken by Cs-corrected STEM (JEM-ARM200F, JEOL) with $80 \mathrm{keV}$ electron beam. PES and X-ray photoemission spectra were measured using the synchrotron source at the $4 \mathrm{D}$ beam-line and NEXAFS spectra were obtained at the $2 \mathrm{~A}$ beamline at Pohang Accelerator Laboratory. The NEXAFS spectra were obtained with partial electron yield at a $55^{\circ}$ angle of incidence of the synchrotron photon beam with respect to the surface of the samples.

Data availability. The data that support the findings of this study are available within the article and its Supplementary Information files, or available from the authors upon request.

\section{References}

1. Novoselov, K. S. et al. Electrical field effect in atomically thin carbon films. Science 306, 666-669 (2004).

2. Zhang, Y., Tan, Y., Stormer, H. L. \& Kim, P. Experimental observation of the quantum Hall effect and Berry's phase in graphene. Nature 438, 201-204 (2005)
3. Novoselov, K. S. et al. Two-dimensional gas of massless Dirac fermions in graphene. Nature 438, 197-200 (2005).

4. Geim, A. K. \& Novoselov, K. S. The rise of graphene. Nat. Mater. 6, 183-191 (2007).

5. Neto, A. H. C., Guinea, F., Peres, N. M. R., Novoselov, K. S. \& Geim, A. K. The electronic properties of graphene. Rev. Mod. Phys. 81, 109-162 (2009).

6. Schwierz, F. Graphene transistors. Nat. Nanotechnol. 5, 487-496 (2010).

7. Bonaccorso, F., Sun, Z., Hasan, T. \& Ferrari, A. C. Graphene photonics and optoelectronics. Nat. Photonics 4, 611-622 (2010).

8. Novoselov, K. S. et al. A roadmap for graphene. Nature 490, 192-200 (2012).

9. Han, M. Y., Özyilmaz, B., Zhang, Y. \& Kim, P. Energy band-gap engineering of graphene nanoribbons. Phys. Rev. Lett. 98, 206805 (2007).

10. Li, X., Wang, X., Zhang, L., Lee, S. \& Dai, H. Chemically derived, ultrasmooth graphene nanoribbon semiconductors. Science 319, 1229-1232 (2008).

11. Loh, K. P., Bao, Q., Ang, K. P. \& Yang, J. The chemistry of graphene. J. Mater Chem. 20, 2277-2289 (2010).

12. Sofo, J. O., Chaudhari, A. S. \& Barber, G. D. Graphene: a two-dimensional hydrocarbon. Phys. Rev. B 75, 153401 (2007).

13. Gao, H. et al. Band gap tuning of hydrogenated graphene: $\mathrm{H}$ coverage and configuration dependence. J. Phys. Chem. C 115, 3236-3242 (2011).

14. Elias, D. C. et al. Control of graphene's properties by reversible hydrogenation: evidence for graphane. Science 323, 610-613 (2009).

15. Ryu, S. et al. Reversible basal plane hydrogenation of graphene. Nano Lett. 8 , 4597-4602 (2008).

16. Balakrishnan, J. et al. Colossal enhancement of spin-orbit coupling in weakly hydrogenated graphene. Nat. Phys. 9, 284-287 (2013).

17. Balog, R. et al. Atomic hydrogen adsorbate structures on graphene. J. Am. Chem. Soc. 131, 8744-8745 (2009).

18. Zhang, Y. et al. Direct observation of a widely tunable bandgap in bilayer graphene. Nature 459, 820-823 (2009).

19. Balog, R. et al. Bandgap opening in graphene induced by patterned hydrogen adsorption. Nat. Mater. 9, 315-319 (2010).

20. Kim, K. S. et al. Large-scale pattern growth of graphene films for stretchable transparent electrodes. Nature 457, 706-710 (2009).

21. Bae, S. et al. Roll-to-roll production of 30-inch graphene films for transparent electrodes. Nat. Nanotechnol. 5, 574-578 (2010).

22. Schultz, B. J. et al. Imaging local electronic corrugations and doped regions in graphene. Nat. Commun. 2, 372 (2011)

23. Avsar, A. et al. Toward wafer scale fabrication of graphene based spin valve devices. Nano Lett. 11, 2363-2368 (2011)

24. Chae, D. et al. Excitonic Fano resonance in free-standing graphene. Nano Lett. 11, 1379-1382 (2011).

25. Ferrari, A. C. Raman spectroscopy of graphene and graphite: disorder, electron-phonon coupling, doping and nonadiabatic effects. Solid State Commun. 143, 47-57 (2007).

26. Banhart, F., Kotakoski, J. \& Krasheninnikov, A. V. Structural defects in graphene. ACS Nano 5, 26-41 (2011).

27. Cançado, L. G. et al. Quantifying defects in graphene via Raman spectroscopy at different excitation energies. Nano Lett. 11, 3190-3196 (2011).

28. Eckmann, A. et al. Probing the nature of defects in graphene by Raman spectroscopy. Nano Lett. 12, 3925-3930 (2012).

29. Smith, R. A. Semiconductors 2nd edn (Cambridge Univ. Press, 1978).

30. Venugopal, G., Krishnamoorthy, K., Mohan, R. \& Kim, S. An investigation of the electrical transport properties of graphene-oxide thin films. Mater. Chem. Phys. 132, 29-33 (2012).

31. Haberer, D. et al. Evidence for a new two-dimensional $\mathrm{C}_{4} \mathrm{H}$-type polymer based on hydrogenated graphene. Adv. Mater. 23, 4497-4503 (2011).

32. Li, Y. \& Chen, Z. Patterned partially hydrogenated graphene $\left(\mathrm{C}_{4} \mathrm{H}\right)$ and its one-dimensional analogues: A computational study. J. Phys. Chem. C 116, 4526-4534 (2012).

33. Ma, Y. et al. Electronic and magnetic properties of the two-dimensional $\mathrm{C}_{4} \mathrm{H}$-type polymer with strain effects, intrinsic defects and foreign atom substitutions. Phys. Chem. Chem. Phys. 14, 3651-3658 (2012).

34. Cheng, S.-H. et al. Reversible fluorination of graphene: Evidence of a twodimensional wide bandgap semiconductor. Phys. Rev. B. 81, 205435 (2010).

35. Morkoç, H. et al. Large-band-gap SiC, III-V nitride, and II-VI ZnSe-based semiconductor device technologies. J. Appl. Phys. 76, 1363-1398 (1994).

36. Wang, J., Zhao, R., Yang, M., Liu, Z. \& Liu, Z. Inverse relationship between carrier mobility and bandgap in graphene. J. Chem. Phys. 138, 084701 (2013)

37. Zhu, Y. et al. Graphene and graphene oxide: Synthesis, properties, and applications. Adv. Mater. 22, 3906-3924 (2010)

38. Yan, L. et al. Chemistry and physics of a single atomic layer: strategies and challenges for functionalization of graphene and graphene-based materials. Chem. Soc. Rev. 41, 97-114 (2012).

\section{Acknowledgements}

We are grateful to Dr Evelin Sullivan and Mr Minseok Kim for discussions. We also thank Dr Sung-pyo Cho and Mr Kyumin Lee for their support in observing microscopic 
images by transmission electron microscopy, Dr Kyuwook Ihm for discussions about PES measurements at PAL (4D beamline), Dr Kyung-Hwa Yoo and Miss Nalae Han for support in e-beam lithography, and Dr Hyunchul Son, Dr Yoshihiro Iwasa and Mr Yu Saito for measurements of properties of FETs. This research was supported in part by Grant (NRF-2014R1A2A1A11050290) and Creative Materials Discovery Program (2015M3D1A1070465) through the National Research Foundation of Korea (NRF) funded by the Ministry of Science, ICT and Future Planning (MSIP), and by Research Program (10044723) funded by the Ministry of Trade, Industry \& Energy of Korean government and the Korea Semiconductor Research Consortium for the development of the future semiconductor device. Experiments at PLS-II were supported in part by MSIP and POSTECH. H.-K.L. acknowledges support from the Basic Science Research through the NRF funded by the Ministry of Education (NRF-2011-0006744). B.H.H. acknowledges financial support from the Global Frontier Research Program (2011-0031629). J.H.K. acknowledges financial support from Grant (2012M3A7B4049802) through the NRF funded by the Korean government (MSIP).

\section{Author contributions}

J.S. and J.H. conceived and designed the study. J.S. fabricated samples and carried out experiments under the guidance of J.H. with the help of the other authors. S.J.K. and B.H.H. prepared the CVD graphene for hydrogenation. B.C.P. and J.H.K. performed the optical absorption experiments and data analyses. H.-K.L. helped to carry out the NEXAFS experiments and to interpret the results. All authors contributed to the discussion of the work. J.H. wrote the manuscript.

\section{Additional information}

Supplementary Information accompanies this paper at http://www.nature.com/ naturecommunications

Competing financial interests: The authors declare no competing financial interests.

Reprints and permission information is available online at http://npg.nature.com/ reprintsandpermissions/

How to cite this article: Son, J. et al. Hydrogenated monolayer graphene with reversible and tunable wide band gap and its field-effect transistor. Nat. Commun. 7, 13261 doi: 10.1038/ncomms13261 (2016).

Publisher's note: Springer Nature remains neutral with regard to jurisdictional claims in published maps and institutional affiliations.

(c) (i) This work is licensed under a Creative Commons Attribution 4.0 International License. The images or other third party material in this article are included in the article's Creative Commons license, unless indicated otherwise in the credit line; if the material is not included under the Creative Commons license, users will need to obtain permission from the license holder to reproduce the material. To view a copy of this license, visit http://creativecommons.org/licenses/by/4.0/

(C) The Author(s) 2016 